\title{
Protée
}

\section{Le spectre de la parenté}

\section{Images génériques et politique de l’hérédité d'après Francis}

\section{Galton}

\section{Alexis Lussier}

Volume 37, numéro 1, printemps 2009

Corps photographiques / Corps politiques

URI : https://id.erudit.org/iderudit/001355ar

DOI : https://doi.org/10.7202/001355ar

Aller au sommaire du numéro

\section{Éditeur(s)}

Département des arts et lettres - Université du Québec à Chicoutimi

ISSN

0300-3523 (imprimé)

1708-2307 (numérique)

Découvrir la revue

Citer cet article

Lussier, A. (2009). Le spectre de la parenté : images génériques et politique de l'hérédité d'après Francis Galton. Protée, 37(1), 59-66.

https://doi.org/10.7202/001355ar

\section{Résumé de l'article}

Les « portraits composites » de Francis Galton (1822-1911) consistaient à surimposer, les uns sur les autres, plusieurs portraits pour dégager, selon lui, le " type » du visage criminel avant " que les traits du visage soient brutalisés par le crime ". Que serait donc un visage criminel auquel il ne manque plus que le crime ? Position d'autant plus étonnante que Galton finit par admettre que l'infamie du visage réside dans chacun des visages photographiés et que l'humanité n'appartient qu'au " type " qui, en l'occurrence, n'existe que par la photographie et dans la photographie. Il s'agit dans cet article de reprendre, à travers une lecture de certains des textes que Galton a consacrés à la photographie, une théorie spécifique de l'image qui repose essentiellement sur un discours moral et politique touchant à l'hérédité des familles. 


\title{
LE SPECTRE DE LA PARENTÉ IMAGES GÉNÉRIQUES ET POLITIQUE DE L'HÉRÉDITÉ D'APRÈS FRANCIS GALTON
}

AlEXIS LUSSIER

\begin{abstract}
Sortir de son halo l'objet en détruisant son aura, c'est la marque d'une perception dont «le sens du semblable dans le monde» se voit intensifié à tel point que, moyennant la reproduction, elle parvient à standardiser l'unique. Ainsi se manifeste dans le domaine de la réceptivité ce qui déjà, dans le domaine de la théorie, fait l'importance toujours croissante de la statistique. (Benjamin, [1936] 1991: 183)
\end{abstract}

Dans son ouvrage Inquiries into Human Faculty and Its Development, Sir Francis Galton (1822-1911), petit cousin de Charles Darwin et lui aussi scientifique de renom, associait le corps criminel, tel qu'il est révélé par la photographie dans la recherche du criminel «type» en Angleterre, à une essentielle "défiguration»: "C'est malheureusement un fait, écrivait Galton, que passablement de criminels typiquement distincts ont fait naître la vérité sur leur espèce, qui est la défiguration la plus triste de la civilisation moderne» ([1907] 1973: 10-11; notre traduction) ${ }^{1}$. C'était déjà dire deux choses. D'abord, le corps criminel touche à la sphère de la «civilisation». Les criminels qu'elle abrite la menacent. Ensuite, la vérité sur l'«espèce» criminelle siège dans son aspect. Le type criminel est non pas la simple métaphore du mal, mais l'incarnation de la criminalité elle-même; par conséquent, il est un corps défiguré qui ne peut qu'inquiéter la figure humaine dans son intégrité physique et morale. À prendre Galton au mot, les questions se multiplient. Le corps criminel peut-il porter atteinte à la figure morale que l'homme civilisé se donne de lui-même? Dans quelle mesure y a-t-il menace de dé-figuration? Et jusqu'à quel point le corps criminel devient-il l'autre de la figure humaine?

C'est que l'idée de "figure humaine" n'est pas assurée à elle-même. Vieille histoire. Déjà, chez les Pères de l'Église, ne disait-on pas que «l'homme porte la ressemblance de deux objets opposés »: une ressemblance originaire à la beauté transcendantale de l'image et une ressemblance à la «brute» qui est un instable mouvement de chair et de violence, en somme une monstruosité et une incertitude quant à la forme humaine (voir Grégoire de Nysse, 2002:168)? C'est que la figure de l'anthropos, au sens où l'entendaient les Pères de l'Église, est d'abord une figure brisée, toujours en péril de se défigurer dans la distance même qui l'éloigne d'une image idéale et transcendante. Et, 
étonnamment, les recherches sur l'hérédité criminelle au XIXe siècle se trouvent à rencontrer les présupposés des premiers théologiens, à savoir que toute image de l'homme n'est jamais qu'une image altérée de par la résurgence de la «brute» qui sommeille en lui et qui risque à tout moment d'entamer la souveraineté de l'homme à sa propre figure.

Ce qui dit bien comment la figure humaine tient sur un paradoxe; un conflit qui s'expose au sein même de la ressemblance de l'homme à sa propre forme idéalisée. C'est pourquoi l'on peut lire dans l'énoncé de Galton quelque chose comme une angoisse devant la défiguration d'un corps photographique, dans la mesure où cette angoisse est aussi celle du photographe, qu'il était en partie, alors que le père de l'eugénisme (la science se donnant pour objet d'étude les possibilités d'amélioration de la race) se proposait de dégager non seulement le type du visage criminel, mais aussi le type idéal de la famille moralement saine et forte de son hérédité anglaise. La proposition deviendra politique, parce que, au lieu de simplement photographier le corps criminel ou le corps de l'individu «sain", il s'agira, d'après les vœux de Galton, d'en repasser par la photographie pour réaliser l'image du type idéal à travers les générations, comme s'il fallait, en quelque sorte, anthropomorphiser l'homme à lui-même.

\section{LE CORPS CRIMINEL DANS L'EIL DE LA SCIENCE}

En 1882, le grand criminologue français Alphonse Bertillon systématise l'identification des criminels basée sur l'utilisation de la photographie. Le fichage anthropométrique devait fixer une identité en s'appuyant sur l'individuation du corps (mensurations et signes distinctifs). Au moment où la photographie permet à la science d'espérer en de nouveaux critères d'objectivité, l'utilisation de la fiche signalétique et l'anthropométrisation des «récidivistes" renouvellent les méthodes d'investigation policière. Comme l'a fort bien étudié Christian Phéline (1985), la photographie s'invente alors comme «technologie politique"; elle introduit de nouveaux rapports entre figuration du corps et force publique, art du portrait et enquête judiciaire. D'un côté, la photographie policière participe à des programmes de comparaison statistique sur les «types criminels». D'un autre côté, l'objectivité du procédé photographique s'accompagne d'une véritable fascination pour les signes de la criminalité. Le visage criminel ne peut être qu'un visage stigmatisé, marqué par les signes de Caïn, comme on disait à l'époque.

Les recherches sur le type criminel s'appuyaient en partie sur les thèses de Prosper Lucas qui avait avancé, dans son Traité philosophique et physiologie de l'hérédité naturelle (1847-1850), l'idée que les causes de la criminalité sont à chercher du côté de l'hérédité. Plus tard, le docteur et aliéniste Benedict Morel, dans son Traité des dégénérescences physiques, intellectuelles et morales de l'espèce humaine (1857), introduit l'idée que le destin de la «race» se soutient d'une visée eschatologique fondée sur la répétition d'un «type primitif» vis-à-vis duquel toutes les déviations auraient correspondu à une dégénérescence de la nature. Or, par rapport au «type primitif idéal» de Morel, Cesare Lombroso, auteur à succès de L'Homme criminel (1876), avançait l'hypothèse que la criminalité ellemême devait reposer sur la répétition d'un certain type criminel, dont la découverte aurait permis au regard de la science de lever le voile sur les causes ancestrales de la criminalité. Dans cette optique, le corps criminel apparaissait comme une véritable hantise. Les marques de Caïn se donnaient à lire dans les corps. Ainsi, lorsque Lombroso revient sur les circonstances de ses découvertes, il soutient que l'analyse phrénologique du crâne d'un individu incontestablement criminel révèle que sa constitution anatomique n'était autre que la reformation contemporaine d'une ossature primitive, conférant à l'homme criminel un statut mi-homme mi-bête dont il aurait été rien de moins que la résurgence:

[...] comme apparaît une large plaine sous l'horizon

enflammé, le problème de la nature et de l'origine

du criminel m'apparut résolu: les caractères des hommes primitifs et des animaux inférieurs devaient se reproduire de nos temps. (Darmon, 1989: 33)

Par conséquent, la criminalité est à comprendre non plus comme au siècle des Lumières, selon le postulat du libre arbitre, mais d'après une hérédité fatale qui situe le criminel dans l'ordre 
des générations. Dans la mouvance de la nouvelle criminologie, Francis Galton devient l'instigateur d'un eugénisme autoritaire, qui présumait enrayer naturellement la criminalité, en employant des méthodes gestionnaires visant la reproduction de l'espèce en fonction des différences et des compatibilités héréditaires (souvent associées aux classes sociales). L'eugénisme de Galton reconduisait en ce sens des programmes fortement appuyés par des politiques répressives, dont les enjeux, qui pouvaient, à la limite, se rapporter à un problème de santé sociale, pointaient vers les risques annoncés d'une prétendue débâcle héréditaire.

Si la criminalité est de nature héréditaire, le remède est par conséquent à rechercher dans les corps. Et c'est sur les corps, et plus spécifiquement sur la reproduction même des corps, qu'il s'agit d'intervenir. Alors que les médecins, aliénistes et criminologues acquièrent un statut de plus en plus marqué dans les sphères juridiques et policières (Davie, 2004: 13), Galton parle résolument de gestion des naissances, encouragé par des concours d'État en "mérite héréditaire», lesquels devaient être clôturés par des cérémonies publiques pour les gagnants, des mariages promotionnels à l'Abbaye de Westminster et des subventions en argent pour les nouvelles familles sélectionnées (ibid.: 200). Pour les criminels et les individus jugés «inaptes», on parle d'interdit de reproduction et de ségrégation perpétuelle.

La vérité, écrit Galton, semble être que la population

criminelle reçoit constamment de nouveaux membres fournis

par des classes qui, sans avoir des caractères criminels très accentués, appartiennent cependant à une partie de l'humanité complètement incapable de jouer un rôle respectable dans notre civilisation moderne, mais parfaitement douée pour se développer dans l'état de demi-sauvagerie, étant naturellement vigoureuse et féconde. De tels individus sont enclins au mal; leurs filles s'unissent à des criminels et donnent le jour à des criminels. (1877: 497)

Tout cela traduit quelque chose comme un malaise dans la civilisation... Qu'est-ce donc que le corps criminel? Une survivance qui est en fait une dégénérescence, et une défiguration, avec la folie et l'épilepsie, comme Galton le dira ailleurs, qui ne peut que mettre en danger la figure idéale de l'espèce que l'eugénisme, par ailleurs, cherchait à restaurer. Le corps criminel est dès lors pris dans une généalogie fatale: il est déterminé dans les formes (aspect et physionomie du visage) par un modèle anté-historique (la brute ancestrale), alors qu'il est perçu comme une criminalité en puissance dont le visage porterait les indices. Photographier le spectre de la parenté criminelle, cela revenait ni plus ni moins à se donner une image, dit Galton, du «type du visage susceptible de tendances criminels, avant (si je peux me permettre cette expression) que le visage ne soit brutalisé par le crime» (1879b: 162; notre traduction) ${ }^{2}$.

\section{IMAGE GÉNÉRIQUE ET PORTRAITS COMPOSITES}

Wittgenstein disait que la "probabilité d'une hypothèse trouve sa mesure dans le degré d'évidence qui est nécessaire pour la rendre intéressante ou pour l'écarter» (1980: 290). Ici, il s'agit non plus de mettre une hypothèse à l'épreuve du réel, mais bien de mettre le réel à l'épreuve d'une hypothèse avant même qu'elle ne perde sa valeur d'évidence. Après Lucas, Morel et Lombroso, la théorie du criminel né va donner dans l'utilisation de la photographie l'espoir de se confirmer. Il s'agit non plus seulement d'en passer par la question de l'hérédité, mais de littéralement faire apparaître le type criminel à travers le spectre de la parenté.

L'expression "image générique» nous est suggérée dans ce contexte par Galton qui désigne une catégorie d'image réalisée en photographie par la superposition, sur une même plaque sensible, de plusieurs portraits d'égales dimensions et de physionomies équivalentes, laissant apparaître, par un effet de coalescence, un portrait étonnamment réaliste et tout à fait saisissant d'un point de vue esthétique ${ }^{3}$. En superposant plusieurs visages, on obtenait un authentique portrait collectif, ou plus exactement, selon les propres termes de Galton, un "portrait composite», ou "portrait générique», produit par impressions successives. Le procédé, qui fut très tôt appliqué aux portraits judiciaires de criminels notoires, permettait de supposer que l'image générique, établie par la photographie, non seulement révélait l'image d'un «genre», un «type», mais donnait à lire in effigie la 
ressemblance commune à tous les visages superposés. En ce sens, le visage criminel souvent décrit par Galton, qui ne cache pas son dégoût et une profonde exaspération, n'était autre chose que le site d'une image qui se surimprime d'une génération à l'autre, et que seule la production des portraits composites aurait permis de rendre visible. Contrairement au bertillonnage, les portraits composites de Galton visaient la reconnaissance d'une appartenance de l'individu à une classe commune dans laquelle se noyaient toutes les singularités. À la mesure anthropométrique du visage, Galton substituait la construction statistique d'un visage-synthèse.

Tout se passe donc, pour Galton, comme si la photographie avait eu les moyens techniques de réaliser, sur la somme des visages, la moyenne de leur ressemblance. Or, cette moyenne obtenue par la photographie se donne sur un mode spécifiquement visuel; c'est une moyenne qui s'actualise, pourrait-on dire, en image. Il ne s'agit donc pas tout à fait de corps photographiés, mais bien d'un corps photographique dont chacun des composants n'est lui-même qu'un visage vague et diaphane qui se précise par surimpression. "Les contours les plus nets et les plus foncés sont partagés par le plus grand nombre de portraits individuels», explique Galton; «les traits du visage purement individuel laissent peu ou pas de trace»; aussi, dans la recherche de la ressemblance la plus commune, les «traits", poursuit Galton, «sont nécessairement répartis d'une manière égale autour de la moyenne, le contour du cliché composite étant la moyenne de tous les composants» (1879a: 134; notre traduction) $)^{4}$.

Le visage obtenu dans le composite apparaît dans un halo de traits flous que Galton se propose de porter à l'examen: on parle alors de bandes et de contours, on s'interroge devant l'ampleur des ombres et la détermination du plus clair et du plus distinct. Ainsi, selon Galton, les contours du visage forment une "bande» et non «une ligne fine»:

La bande sera plus sombre en son milieu chaque fois que

les portraits composants ont le même type de physionomie, et la largeur ou le flou de cette bande sera une indication

de l'envergure de la déviation des portraits composants de type commun. (Ibid.; notre traduction) ${ }^{5}$
Il s'agit pour le moins d'un étonnant calcul qui combine le désir de mesurer au désir de voir (Sekula, 1986: 44). Le portrait, une fois réalisé selon une technique que Galton a souvent eu l'occasion de décrire (enlignement des visages, temps d'exposition, etc.), était en quelque sorte entrepris par un regard censément analytique, alors que l'aspect du composite est lu à la façon d'une compilation de données («cumulative result» [Galton, 1973: 132]) - comme si on avait sous les yeux la transcription visuelle d'un résultat statistique susceptible de rendre visible ce qui est commun, en laissant s'estomper ce qui est individuel.

En ce sens, la description même du portrait composite comporte une appréciation morale et politique: ne sera déterminé et convergent que ce qui est commun, tandis que tout ce qui est individuel et déviant sera flou et indéterminé. Telle était l'hypothèse qu'il a fallu mettre à l'épreuve de la photographie. Cette hypothèse est vite devenue dans l'esprit de Galton une évidence, voire une certitude. Comment, en effet, ne pas voir dans le portrait composite une authentique somme des visages, puisque la résultante contient les traits de tous les visages surimprimés en une seule et même image? C'est pourquoi, bien plus que d'une simple moyenne comme Galton le défendra luimême, il s'agit de la jonction photographique des traits convergents laissant apparaître une sorte de canevas de la "commune mesure» des visages.

Les portraits composites sont, par conséquent, bien plus que des moyennes, parce qu'ils incluent les visages de tous les individus qui les composent. Ils sont un équivalent pictural de ces tableaux statistiques d'où sont tirées les moyennes. Il n'y a pas d'exemples plus parfaits auxquels ils peuvent s'accorder, que ce que les métaphysiciens entendent par généralisations, quand les objets généralisés sont objets de visions, et quand ils appartiennent au même groupe, dont l'une des caractéristiques importantes est que les traits médians peuvent être de loin plus fréquents que ceux qui en divergent. (Galton, 1879b: 163; notre traduction) ${ }^{6}$

En 1877, Galton avait tout d'abord imaginé un dispositif spéculaire pour multiplier, à l'usage de la photographie, les points de vue d'un même corps, placé à égale distance de trois miroirs 
périphériques, afin de saisir le "portrait direct» d'un sujet d'après quatre angles simultanés:

Le résultat serait une photographie ordinaire du sujet, entourée de trois vues différentes de sa tête. Une échelle graduée fixée au cadre serait aussi photographiée, et fournirait des moyens de mesure exacts. (1877: 496)

Le système du portrait en miroir ne faisait encore que multiplier les points de vue en fonction d'une optique tout aussi précise qu'irréfutable sur le plan anthropométrique. Par contre, de façon beaucoup plus étonnante et, dans une certaine mesure, de façon beaucoup plus radicale, la technique des «portraits composites» qu'il développait à la même époque déplaçait considérablement tout point de vue. Le portrait type n'était que la production d'une fiction de visage (Galton dit lui-même qu'il s'agit de portraits imaginaires) mais, en réalisant les «impressions successives de ses images constituantes", il produisait en même temps un modèle ayant statut de référence.

Or, c'est précisément sur ce point qu'il convient d'insister. Si la photographie permet de dégager un type de référence, l'enjeu fondamental porte sur la ressemblance de l'individu à la référence elle-même. En ce sens, le statut de cette image que Galton cherche à employer n'est pas de l'ordre d'une pure scientificité. Elle appartient à des préoccupations beaucoup plus profondes et infiniment plus difficiles à circonscrire qui touchent aux objectifs de la typologie photographique comme aux fins de la ressemblance.

\section{LES FINS DE LA RESSEMBLANCE}

Lorsque j'ajuste les portraits pour en faire un composite, et au moment où l'ajustement devient effectif, je fais toujours l'expérience d'un vif sentiment de satisfaction curieusement analogue à la première reconnaissance d'une ressemblance incertaine. J'ai le même sentiment désagréable devant un casse-tête que je ne peux terminer, accompagné de la conviction que ce casse-tête est sur le point d'être résolu. L'instant suivant, la coalescence prend place entre ce qui a été vu et ce qui a été rassemblé. Je suis sûr, autant que cela se peut, qu'il est possible d'établir que l'analogie entre la capture de la coincidence de deux portraits similaires superposés par l'optique et la coïncidence d'un objet visible avec une impression passée ou une idée générale préexistante est vraie et non pas seulement métaphorique.

(Galton, 1879b: 164; notre traduction) ${ }^{7}$

En évoquant les photographies de Galton, Georges Bataille sera plus tard sensible au fait que les portraits composites reprenaient la recherche classique de l'image idéale comme site de la figuration du corps, pour critiquer cette assurance en la réalité naturelle de cette idée générale préexistante (1970: 228-230). En effet, parallèlement aux portraits composites de criminels ou de malades chroniques, Galton poursuivait le projet d'établir, avec la même technique, la ressemblance familiale idéale entre plusieurs membres d'une même famille, voire à partir de plusieurs portraits d'une même personne afin de fixer dans la succession la ressemblance «la plus probable» de cette personne. Une image idéale, parce que non naturelle, c'est-à-dire qui ne revient à aucun corps en particulier, mais une image que l'on dira plus belle, parce que non entamée par les accidents du corps. En ce sens, on peut remarquer avec Bataille combien les composites de Galton semblaient réhabiliter "l'idée platonicienne" comme forme idéale et critère de beauté. Bien que le composite soit encore un visage imaginaire, il s'avérait, dit Galton, plus humain et plus beau que chacun des visages photographiés. C'est que l'humanité, entendue elle-même comme catégorie idéale, est dans le «type» qui n'existe que dans la photographie et par la photographie. Toute déviance quant à la moyenne est un risque de dégénérescence ou de «médiocrité», selon les termes de Galton.

L'image composite, écrit Bataille, donnerait ainsi une sorte de réalité à l'idée platonicienne, nécessairement belle. En même temps la beauté serait à la merci d'une défnition aussi classique que celle de la commune mesure.

Mais chaque forme individuelle échappe à cette commune mesure et, à quelque degré, est un monstre. (Ibid.: 230)

On sait qu'en 1886 Gabriel Tarde souhaitait «opposer [la silhouette criminelle] au type idéal de la beauté humaine» (Saurisse, 1997: 73). Dans le même ordre d'idées, et en contrepartie des portraits de criminels types, Galton aura cherché à établir une «forme idéale typique» (ou «type central») produite 
à partir des portraits des officiers d'élite des Royal Engineers, dont le visage lui inspirait spontanément vigueur, résolution, intelligence et franchise.

Ce visage et les qualités qu'il connote nous donnent probablement un indice de la direction dans laquelle le stock de la race anglaise devrait progresser. C'est la notion essentielle de race qui peut nous donner une certaine forme idéale typique à partir de laquelle les individus peuvent dévier dans toutes les directions, mais aussi, et surtout, où ils peuvent se rassembler et à travers laquelle leurs descendants vont continuer à se rassembler. Il est nécessaire de n'encourager, autant que faire se peut, l'accouplement que de ceux qui sont les plus conformes au type central et de restreindre autant que possible l'accouplement de ceux qui en dévient largement. (Galton, 1973: 10; notre traduction) ${ }^{8}$

Nous voici donc au terme politique de l'entreprise. Il faudrait garder la race à l'intérieur de son image idéale typique, une image générique de référence, pour éviter une dispersion des corps au nom de la commune mesure. Galton, on le sait, faisait mal la distinction entre hérédité et classe sociale. Or, cette proposition est politique, dans la mesure où elle superpose classe sociale et "classe héréditaire» (selon une hérédité forte et centrale ou faible et déviante) au nom d'un rassemblement, lequel devrait, d'après Galton, être réalisé autour de la commune mesure donnée pour référence. Sous le couvert d'un alibi scientifique, la photographie vise une fin politique dans la mesure où l'image générique, établie par la photographie, doit participer à une véritable économie de l'hérédité au sein de l'oïkos, de la demeure.

Ainsi, on peut reconnaître dans l'entreprise des portraits composites une double intention, dont la seconde constitue une appropriation de la première. Dans un premier temps, il fallait faire se dissoudre dans l'image photographique les singularités des visages individuels pour obtenir une image générique; mais, dans un second temps, il s'agissait de repartir de la forme généralisée pour rassembler les singularités à l'intérieur d'une image photographique de référence. Cette image, invisible à l'oeil nu, dit Galton, pouvait dès lors être élevée au rang de type originaire.

À cet égard, la figure humaine qui est ici ramenée sous la catégorie idéale du type aurait consisté, selon les thèses de Galton, à faire en sorte que l'homme se reproduise à l'intérieur d'une commune mesure dont la "forme idéale typique» est le centre. Conséquemment, la photographie devenait le témoin de l'intégrité des corps dans le champ d'une référence accréditée qui devait participer, dirait Pierre Legendre, aux «montages de l'identité» nécessaires à "la reproduction de l'homme comme image de l'homme» (1994: 38). L'énoncé ici n'est en rien tautologique, si l'on considère qu'entre l'homme et son image subsiste une essentielle division qu'il s'agit précisément de colmater par l'institution même de la figure et par la gestion de la reproduction. Division entre le corps criminel et le corps moralement sain de l'homme du commun; division aussi entre l'homme du commun et sa propre figuration idéale. C'est qu'en cherchant à restaurer un modèle eugénique de référence, la photographie de la forme idéale typique produisait deux références distinctes et mutuellement opposées: d'une part, un portrait de la référence commune; d'autre part, un portrait de la référence déviante, selon laquelle le corps criminel n'est jamais que distance et altération du corps humain à l'égard du type central. À tout prendre, Galton se proposait de dégager un type de visage à partir de ce qu'il prenait déjà lui-même pour un visage typiquement criminel. Comme l'écrivait Hegel autrefois:

C'est penser abstraitement que de ne rien voir dans l'assassin que cet abstrait qu'il est un assassin, en annihilant par cette qualité simple tout autre trait humain en lui.

([1807] 1983: 49)

Or, Galton allait en partie renverser cette position. Si la photographie composite ne peut réaliser qu'une abstraction, c'est cette certitude en la vérité de l'abstraction qui fait dire à Galton que la vérité n'est visible que dans le type et non dans le corps réel de l'homme. C'est bien pourquoi le corps photographique n'est jamais ici la preuve naturelle de l'existence d'un type criminel, puisque les «marques de Caïn» disparaissent dans le composite pour ne laisser que des «types de visage».

Les marques de Caïn sont variées; donc, les traits particuliers de chaque criminel ne se renforcent pas dans le composite, mais disparaissent. Il ne reste que des types de visages auxquels 
s'ajoutent certaines des nombreuses marques de Caïn qui sont destinées à se fixer. (1879b: 162; notre traduction) ${ }^{9}$

Que peut bien être un visage criminel auquel il ne manque plus que le crime?

La technique donne un visage au caractère minable, mais sans infamie évidente. Les visages individuels sont bien infâmes, mais infâmes de différentes façons; quand ils sont combinés, les particularités individuelles disparaissent et l'humanité commune d'un type inférieur est tout ce qui en ressort.

(Galton, 1973: 11; notre traduction) ${ }^{10}$

C'est bien le sens donné à cette image, plutôt que son objectivité scientifique, qui pose problème. En fin de compte, Galton ne faisait que surimprimer, l'un sur l'autre, des portraits de criminels, donc déjà suspects de porter en eux l'image abstraite du criminel type, alors que l'effet photographique de l'image générique avait pour résultat, toujours selon Galton, de repousser la représentation du corps criminel dans les limites indéfinies d'un «type inférieur». «L'échec, comme le dit Pierre Saurisse, est maquillé par le flou des conclusions" (1997: 72).

Ce faisant, le type criminel risquait de n'être qu'une référence sans contenu, à tout le moins sans contenu visuel, comme si les portraits composites avaient flotté un instant sur une ambiguité de la référence à laquelle la photographie aurait dû répondre en supportant le fantasme de réinsérer l'image humaine au centre civilisateur de sa propre figure.

\section{POLITIQUE DE LA RÉFÉRENCE}

Bien qu'il s'agisse d'abord d'un usage scientifique de la photographie, la construction de la référence idéale, telle qu'on la voit survenir au sein de la société qui se met en place au XIXe siècle, et telle que Foucault entreprit de la décrire dans Surveiller et punir, ne touche pas moins à l'organisation politique des corps. Dans l'espace avéré impur de la société moderne, non seulement l'établissement du type supportait «un même programme de contrôle du mal où stratégies du pouvoir et du savoir se confondent» (Saurisse, 1997: 73), mais la photographie était mise au service d'une vérité naturelle de la figure humaine, entendue comme figure typologique, en vertu de laquelle devaient se mettre en place des politiques gestionnaires, touchant à l'organisation sociale de l'hérédité. Mais ce qu'il y a de plus étonnant, c'est que seule la photographie composite est donnée par Galton comme le plus sûr moyen de connaitre cette image tout en construisant photographiquement la référence destinée à l'homme du commun. Autant dire que la photographie aurait permis ni plus ni moins que de rendre visible, à l'échelle de la "commune mesure", l'image invisible de la référence, pour préserver du même coup l'image de la race d'une potentielle défiguration, et de la perte nécessairement fatale, nécessairement inhumaine, qu'elle entraînerait. Il fallait donc fixer l'image de l'homme à l'intérieur d'une image de synthèse, pour mieux en isoler la figure dans l'espace de la civilisation moderne. Or, fixer l'image n'est pas encore figurer. Pour figurer, il faut d'abord donner forme à un aspect en lui prescrivant une limite, bref, en l'informant; et on peut entendre ici par «informer» ce qu'on entendait anciennement par informare: «représenter idéalement», «former en esprit».

Bien qu'elle ait emprunté à l'esthétique le motif de l'idéal comme critère de beauté, cette entreprise n'était pas délibérément esthétique, mais elle était politique parce qu'elle prétendait, sous le couvert de la science, passer par la photographie des corps pour gérer la reproduction de l'espèce. Rien de moins... Si le corps criminel, qui donc s'oppose au type central selon Galton, est donné comme une menace touchant à l'hérédité des familles, et s'il est susceptible de contaminer par voie de reproduction le «stock" héréditaire de la race, cela dépend de ce qu'on entend par corps criminel, mais aussi de ce qu'on entend faire de la représentation même du criminel établie par la photographie. Par conséquent, introduire la dimension politique du corps en photographie ne consiste pas seulement à nous interroger sur la représentation $d u$ politique en photographie, ou sur les enjeux politiques de la représentation du corps, mais nous force à envisager une politique des emplois et des fins de la photographie, tant il est vrai, comme disait Benjamin, que dans «la représentation de l'image de l'homme par l'appareil, l'aliénation de l'homme par lui-même trouve une utilisation hautement productive» (1991: 200). 
À la fin, qu'en est-il du corps photographique? Le corps criminel y devient corps politique parce que la photographie ne se contente pas de donner une image du corps, mais établit une solution de continuité entre l'homme et sa référence imaginaire dans l'espace social. Et bien que l'image générique ne soit plus théoriquement une image du corps, il s'agissait de retourner au corps pour décider de son destin, quand le procès de la ressemblance, d'un corps à sa figuration standardisée, se joint à une politique de la référence.

\section{NOTES}

1. "It is unhappily a fact that fairly distinct types of criminals breeding true to their kind have become established, and are one of the saddest disfigurements of modern civilisation". 2. "They are instructive as showing the type of face that is apt to accompany criminal tendencies, before (if I may be allowed the expression) the features have become brutalised by crime".

3. Galton eut souvent l'occasion d'exposer en détail la méthode des portraits composites; on peut cependant retenir deux articles essentiels (1879a et 1879b). Ces articles sont tous deux repris en partie dans son ouvrage Inquiries into Human Faculty and Its Development ([1907] 1973). 4. "Those of its outlines are sharpest and darkest that are common to the largest number of the components; the purely individual peculiarities leave little or no visible trace. The latter being necessarily disposed equally on both sides of the average, the outline of the composite is the average of all the components". 5. "It is a band and not a fine line [...]. The band will be darkest in its middle whenever the component portraits have the same general type of features, and its breadth, or amount of blur, will measure the tendency of the components to deviate from the common type". 6. "Composite portraits are, therefore, much more than averages, because they include the features of every individual of whom they are composed. They are the pictorial equivalents of those elaborate statistical tables out of which averages are deduced. There cannot be a more perfect example than they afford, of what the metaphysicians mean by generalisations, when the objects generalised are objects of vision, and when they belong to the same typical group, one important characteristic of which is that medium characteristics should be far more frequent than divergent ones ".

7. "When I am adjusting portraits to make a composite, and at the moment when the adjustment is being effected, I always experience a quick sense of satisfaction curiously analogous to that which is felt on the first recognition of a doubtful likeness of any kind. I have the same disagreeable feeling of the existence of a puzzle, which I cannot make out, accompanied by the conviction that the puzzle is on the point of being solved. In the next instant coalescence takes place between what is seen and what was recollected. I am as sure as it is possible to be on such grounds as these, that the analogy between catching the coincidence of two similar portraits when optically superposed, and that of the coincidence of a visible object with a past impression or with a pre-existent general idea, is true and not metaphorical only". 8. "This face and the qualities it connotes probably gives a clue to the direction in which the stock of the English race might most easily be improved. It is the essential notion of a race that there should be some ideal typical form from which the individuals may deviate in all directions, but about which they chiefly cluster, and towards which their descendants will continue to cluster. The easiest direction in which a race can be improved is towards that central type, because nothing new has to be sought out. It is only necessary to encourage as far as practicable the breed of those who conform most nearly to the central type, and to restrain as far as may be the breed of those who deviate widely from it ". 9. «The brands of Cain are varied; therefore the special expressions of different criminals do not reinforce one another in the composite, but disappear. What remain are types of faces on which some one of the many brands of Cain is frequently destined to be set ". 10. «I have made numerous composites of various groups of convicts, which are interesting negatively rather than positively. They produce faces of a mean description, with no villainy written on them. The individual faces are villainous enough, but they are villainous in different ways, and when they are combined, the individual peculiarities disappear, and the common humanity of a low type is all that is left ».

\section{RÉFÉRENCES BIBLIOGRAPHIQUES}

BATAILLE, G. [(1930) 1970]: «Les écarts de la nature», Euvres complètes, vol. I, Paris, Gallimard, 228-230.

BENJAMIN, W. [(1936) 1991]: "L'œuvre d'art à l'époque de sa reproduction mécanisée ", Écrits français, Paris, Gallimard, coll. «folio », 147-248.

BROGOWSKI, L. [2003]: «De l'idéal (dé)tourné en Witz: La photographie composite de Francis Galton et ses résonances ", Revue d'esthétique, no 43, 153-175.

DARMON, P. [1989]: Médecins et assassins à la Belle Époque, Paris, Seuil. DAVIE, N. [2004]: Les Visages de la criminalité: À la recherche d'une théorie scientifique du criminel type en Angleterre, 1860-1914, Paris, Kimé. FOUCAULT, M. [1975]: Surveiller et punir, Paris, Gallimard. GALTON, F. [1877]: «La psycho-physique», Revue scientifique, vol. 13, 494-498;

[1879a]: «Composite portraits, made by combining those of many different persons into a single resultant figure ", The Journal of the Anthropological Institute of Great Britain and Ireland, vol. 8, 132-144;

[1879b]: "Generic Images ", Nineteenth Century, vol. VI, 157-169;

[1882]: «Photographic Chronicles from Childhood to Age», Fortnightly Review, vol. 31, 26-31;

[(1907) 1973]: Inquiries into Human Faculty and Its Development, New York, AMS Press. GINZBURG, C. [2004]: «Family Resemblances and Family Trees: Two Cognitive Metaphors», Critical Inquiry, no 30, printemps, 537-556. GREEN, D. [1984]: "Veins of Resemblance: Photography en Eugenics", Oxford Art Journal, vol. 7, n 2, 3-16.

GRÉGOIRE DE NYSSE [2002]: La Création de l'homme, trad. de J. Laplace, Paris, Cerf, coll. « Sources chrétiennes».

HEGEL, G. W. F. [(1807) 1983]: «Qui pense abstraitement? », trad. de H. Müller, Ornicar?, no 26-27, 47-50.

LEGENDRE, P. [1994]: Leçons III. Dieu au miroir: Étude sur l'institution des images, Paris, Fayard.

MOREEL, J.-F. [2007]: Le Darwinisme, envers d'une théorie, Paris, Éd.

François-Xavier de Guibert.

PHÉLINE, C. [1985]: L'Image accusatrice, Laplume (France), Association de critique contemporaine en photographie, coll. «Les cahiers de la photographie", no 17 .

SAURISSE, P. [1997]: « Portraits composites: la photographie des types physionomiques à la fin du XIXe siècle», Histoire de l'art, no 37/38, mai, 67-78.

Sekula, A. [1986]: «The Body and the Archive», October, vol. 39, hiver, 3-64.

WitTGENSTEIN, L. [(1969) 1980]: Grammaire philosophique, trad. de M.-A. Lescourret, Paris, Gallimard, coll. «folio». 